\title{
Development of New Metallic Gasket and its Optimum Design for Leakage Performance*
}

\author{
Hasan Aftab SAEED ${ }^{* *}$, Satoshi IZUMI ${ }^{* *}$, Shinsuke SAKAI ${ }^{* *}$, \\ Shigeyuki HARUYAMA ${ }^{* * *}$, Masato NAGAWA ${ }^{* * * *}$ and Hideki NODA ${ }^{* * * *}$ \\ **University of Tokyo, \\ 7-3-1 Hongo, Bunkyo-ku, Tokyo, Japan \\ E-mail: hasan@fml.t.u-tokyo.ac.jp \\ ***Fukuoka Industrial Technology Center, \\ 3-6-1 Norimatsu, Yahata Nishi-ku, Kitakyushu, Japan

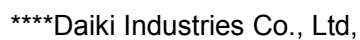 \\ 11-16 Hamamachi, Moji-ku, Kitakyushu, Japan
}

\begin{abstract}
This paper introduces a new all-metal gasket that incorporates strategically located circumferential annular lips that form seal lines with the flanges. This gasket, named Super Seal Gasket, by virtue of its special shape, makes use of the material's spring effect, resulting in sealing performance. This change in approach, from the traditional one based on material development to one of mechanical design, brings with it the need for optimization of the different design parameters for leakage performance. We were able to develop an optimization methodology for this new product using the Taguchi method. As a test case, a 25A sized industrial gasket was optimized using this methodology. In FEM analysis, contact stress and deformation information was used to quantify leaking. Helium leak testing reveals considerable improvement in the sealing performance, hence verifying the applicability of the methodology developed. This work has proved that material development is not the only approach towards the development of new, more effective gaskets and novel gaskets, such as the one introduced in this study, have a useful part to play in the sealing of flanged joints.
\end{abstract}

Key words: Optimization, Robust Design, Finite Element Method, Taguchi Method, Sealing Performance, Asbestos-Free Gaskets, Metal Gaskets

\section{Introduction}

Elimination of asbestos from the Japanese sealing industry is proving to be an elusive goal. Despite government regulation banning all new use by the year 2008, no suitable alternatives are forthcoming ${ }^{(1)}$. To date, most of the research on the part of major gasket manufacturers has been focused on developing new materials with asbestos-like sealing properties, meeting varying degrees of success. While development of materials for sealing purposes is very important and will doubtless meet more success in future; at present, the demand for a viable alternative is mostly unfulfilled.

In this work, we propose a new approach that is distinct from the traditional one based on development of new materials. We introduce Super Seal Gasket ${ }^{(2)}$, a new all-metal gasket that incorporates strategically placed circumferential annular lips. These lips, owing to the spring effect of the metal, form seal lines with flanges. This all important spring effect or elastic response is a function of material properties and physical dimensions of the gasket. Because sealing performance depends on this elastic response, material properties and physical dimensions of the gasket must be optimized for best performance. 
However, gaskets are used in industry under a wide range of environmental and operating conditions. Thus it is imperative that they are designed to work not just in carefully controlled laboratory conditions but also in the rough plant environment. The objective thus, is robust design - which is immune or least sensitive to variations in environmental and operating conditions - as opposed to simple optimized design. Toward that end, and as a test case, we have optimized a 25A sized Super Seal Gasket for sealing performance.

\section{Optimization}

\subsection{Robust design by Taguchi method}

Every engineered system is supposed to perform a specific task in response to a specific input (Fig. 1). A system consists of a combination of design parameters. Noise factors, in the form of variations in operating as well as environmental conditions, have a negative effect on the system, in that they tend to cause variation in output of the system. To achieve robust design, we need to set design parameters so that output is insensitive or least sensitive to noise factors.

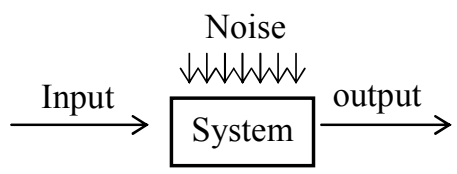

Fig. 1 Engineered system

In Taguchi methodology, this is achieved by introducing noise factors into the design of experiments in a deliberate way. Once quantitative effects of these noise factors on system output are known, it is possible to figure out the most robust configuration.

In the present problem, gasket corresponds to "system" (Fig. 1). This gasket is defined in terms of its design parameters. These design parameters are depicted in Fig. 2, which shows the gasket cross section. These have to be optimized for the most robust sealing performance. The design factors, along with their respective levels are shown in Table 1.

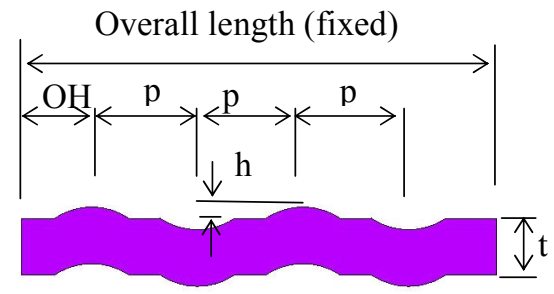

Fig. 2 Gasket cross section and design parameters

Table 1 Design factors

\begin{tabular}{|c|c|c|c|}
\hline Design factor & Level 1 & Level 2 & Level 3 \\
\hline Material (Mat) & Mat 1 & Mat 2 & - \\
\hline Lip height (h) [mm] & 0.2 & 0.3 & 0.4 \\
\hline Lip number (LN) & $2 \times 2$ & $2 \times 1$ & $1 \times 1$ \\
\hline Pitch (p) [mm] & 3 & 4 & 5 \\
\hline Overhang (OH) [mm] & 3 & 4 & 5 \\
\hline Thickness (t) [mm] & 1 & 1.5 & 2 \\
\hline
\end{tabular}

Levels for physical dimensions were selected by engineering judgment, based on the original trial and error design. Level $2 \times 1$ under "Lip number" means two lips at the top 
surface and one lip at the bottom surface of gasket. The two materials were considered as elastic perfectly-plastic materials with $\sigma_{\mathrm{y}} / E$ of $1.8 \times 10^{-3}$ and $2.5 \times 10^{-3}$, the two extremes of ordinary steel materials, where $\sigma_{y}$ is the yield strength and $E$ is the modulus of elasticity (taken as 205GPa). Poisson's ratio $v$ for both were taken as 0.3 . Because we had one design factor with two levels and five design factors with three levels each, we used an $\mathrm{L}_{18}$ inner orthogonal array (number of trial conditions: 18), which can accommodate a maximum of one design factor with two levels and seven design factors with three levels each ${ }^{(3)}$. The $\mathrm{L}_{18}$ inner orthogonal array with design factors and their respective levels is shown in Table 2. The numbers 1, 2, and 3, stand for the levels corresponding to the different design factors.

Table $2 \mathrm{~L}_{18}$ Inner (Design) Orthogonal Array

\begin{tabular}{|c|c|c|c|c|c|c|c|c|c|c|c|c|}
\hline No. & A & B & C & D & E & F & Mat & h & LN & p & OH & t \\
\hline 1 & 1 & 1 & 1 & 1 & 1 & 1 & Mat1 & 0.2 & $2 \times 2$ & 3 & 3 & 1 \\
\hline 2 & 1 & 1 & 2 & 2 & 2 & 2 & Mat1 & 0.2 & $2 \times 1$ & 4 & 4 & 1.5 \\
\hline 3 & 1 & 1 & 3 & 3 & 3 & 3 & Mat1 & 0.2 & $1 \times 1$ & 5 & 5 & 2 \\
\hline 4 & 1 & 2 & 1 & 1 & 2 & 2 & Mat1 & 0.3 & $2 \times 2$ & 3 & 4 & 1.5 \\
\hline 5 & 1 & 2 & 2 & 2 & 3 & 3 & Mat1 & 0.3 & $2 \times 1$ & 4 & 5 & 2 \\
\hline 6 & 1 & 2 & 3 & 3 & 1 & 1 & Mat1 & 0.3 & $1 \times 1$ & 5 & 3 & 1 \\
\hline 7 & 1 & 3 & 1 & 2 & 1 & 3 & Mat1 & 0.4 & $2 \times 2$ & 4 & 3 & 2 \\
\hline 8 & 1 & 3 & 2 & 3 & 2 & 1 & Mat1 & 0.4 & $2 \times 1$ & 5 & 4 & 1 \\
\hline 9 & 1 & 3 & 3 & 1 & 3 & 2 & Mat1 & 0.4 & $1 \times 1$ & 3 & 5 & 1.5 \\
\hline 10 & 2 & 1 & 1 & 3 & 3 & 2 & Mat2 & 0.2 & $2 \times 2$ & 5 & 5 & 1.5 \\
\hline 11 & 2 & 1 & 2 & 1 & 1 & 3 & Mat2 & 0.2 & $2 \times 1$ & 3 & 3 & 2 \\
\hline 12 & 2 & 1 & 3 & 2 & 2 & 1 & Mat2 & 0.2 & $1 \times 1$ & 4 & 4 & 1 \\
\hline 13 & 2 & 2 & 1 & 2 & 3 & 1 & Mat2 & 0.3 & $2 \times 2$ & 4 & 5 & 1 \\
\hline 14 & 2 & 2 & 2 & 3 & 1 & 2 & Mat2 & 0.3 & $2 \times 1$ & 5 & 3 & 1.5 \\
\hline 15 & 2 & 2 & 3 & 1 & 2 & 3 & Mat2 & 0.3 & $1 \times 1$ & 3 & 4 & 2 \\
\hline 16 & 2 & 3 & 1 & 3 & 2 & 3 & Mat2 & 0.4 & $2 \times 2$ & 5 & 4 & 2 \\
\hline 17 & 2 & 3 & 2 & 1 & 3 & 1 & Mat2 & 0.4 & $2 \times 1$ & 3 & 5 & 1 \\
\hline 18 & 2 & 3 & 3 & 2 & 1 & 2 & Mat2 & 0.4 & $1 \times 1$ & 4 & 3 & 1.5 \\
\hline
\end{tabular}

Bolt preload corresponds to "input" (Fig. 1); without satisfactory preload, sealing cannot be achieved. In bolted flanged joints, the most critical noise factor is the variation in this preload. Friction, elastic interactions between joint members, variations in operator behavior, all result in the fact that it is very difficult to control and predict variation in bolt preload; hence to neglect this variation during design would be a mistake. Therefore, variation in preload and variation in internal pressure are taken as the two noise factors having two levels each, as shown in Table 3.

Table 3 Noise factors

\begin{tabular}{|c|c|c|}
\hline Noise factor & $\begin{array}{c}\text { Level 1 } \\
\text { Design level }\end{array}$ & $\begin{array}{c}\text { Level 2 } \\
\text { Unsafe-side }\end{array}$ \\
\hline Bolt preload (PL) $[\mathrm{kN}]$ & 33.4 & 30.0 \\
\hline Internal pressure (IP) $[\mathrm{MPa}]$ & 20 & 22 \\
\hline
\end{tabular}

The unsafe-side figure for bolt preload $(-10 \%)$ is based on empirical observations that suggest that even well maintained (lubricated) flanged joints, closed by effective bolt-up procedures can display variations up to $\pm 10 \%{ }^{(4,5)}$; Whereas that for internal pressure $(+10 \%)$ is chosen arbitrarily. Since we had two noise factors with two levels each, we used an $\mathrm{L}_{4}$ outer orthogonal array (number of trial conditions: 4), which can accommodate a 
maximum of three noise factors with two levels each ${ }^{(3)}$. The $\mathrm{L}_{4}$ outer orthogonal array with noise factors and their respective levels is shown in Table 4 . The numbers 1 and 2 stand for the levels corresponding to the different design factors. Thus in all, 72 finite element simulations specified by the Taguchi method were run.

Table $4 \mathrm{~L}_{4}$ Outer (Noise) Orthogonal Array

\begin{tabular}{|c|c|c|c|c|}
\hline No. & A & B & PL & IP \\
\hline 1 & 1 & 1 & 33.4 & 20.0 \\
\hline 2 & 1 & 2 & 33.4 & 22.0 \\
\hline 3 & 2 & 1 & 30.0 & 20.0 \\
\hline 4 & 2 & 2 & 30.0 & 22.0 \\
\hline
\end{tabular}

Owing to the fact that the physics of this new gasket are not known, it is not at all easy to decide upon the "output" (also called evaluation criterion) of this system (Fig. 1). Noda et al., ${ }^{(6)}$ dealing with a similar metallic gasket have contended that contact stress and a "suitable" plastic zone size are good indicators of sealing performance. The gaskets with plastic zones both smaller and greater than that size perform badly. However, there is no quantitative definition of a suitable plastic zone size. Therefore plastic zone size could not be considered as the evaluation criterion. Instead we selected contact area along with contact stress as the two criteria of evaluation. We supposed that higher values for both of these would result in higher sealing performance (in Taguchi terminology, "bigger is better" quality characteristic in terms of both criteria of evaluation). The reason for selecting the former was that it is reasonable to suppose that a bigger contact area can counter the ill effects of surface roughness. Which one of the two evaluation criteria was more important, was not clear at this stage. Therefore it was decided to carry out the optimization simulations and their analysis in terms of the two criteria independently.

For comparing the performance of the different design configurations, Taguchi method puts emphasis on $\mathrm{SN}$ ratio $(\mathrm{S} / \mathrm{N})$ as opposed to simple average of output. It is so, because in order to achieve robustness, we must consider standard deviation instead of basing our decisions merely on averages. In reliability engineering, coefficient of variation (COV) is used as a measure of quality for the same reason.

$$
\text { COV }=\frac{\sigma}{\mu}
$$

Where, $\sigma$ is the standard deviation, and $\mu$ is the average of output. The lower the COV, the lower is the variation in performance, hence the higher is robustness. Taguchi method uses the same general concept, only takes square of its inverse. The quantity that results is called $\mathrm{S} / \mathrm{N}$; it is the ratio of the performance of the desired function to the variability of that function ${ }^{(7)}$. Naturally, the higher the $\mathrm{S} / \mathrm{N}$, the lower is the variation in performance, and the higher is robustness.

$$
S / N=-10 \log \left(\frac{1}{n} \sum_{n}\left(y_{n}-m\right)^{2}\right)
$$

Where, $n$ is the number of data points, $y_{n}(n=1,2, \ldots, n)$ stands for individual output values, and $m$ is the target output value. This expression applies to "nominal is best" quality characteristic systems; ones with a specified target value. For bigger is better quality characteristic, $\mathrm{S} / \mathrm{N}$ is calculated as follows:

$$
S / N=-10 \log \left(\frac{1}{n} \sum_{n} \frac{1}{y_{n}^{2}}\right)
$$

Since both our evaluation criteria are considered to have "bigger is better" quality 
characteristic, Equation (3) was used to calculate $\mathrm{S} / \mathrm{N}$.

\subsection{FEM model}

Due to limited scope of symmetry boundary conditions of this problem in general and a 25A gasketed flange in particular, fully 3D meshes resulted in extremely computationally expensive solutions, especially considering the fact that we had to run 72 simulation runs.

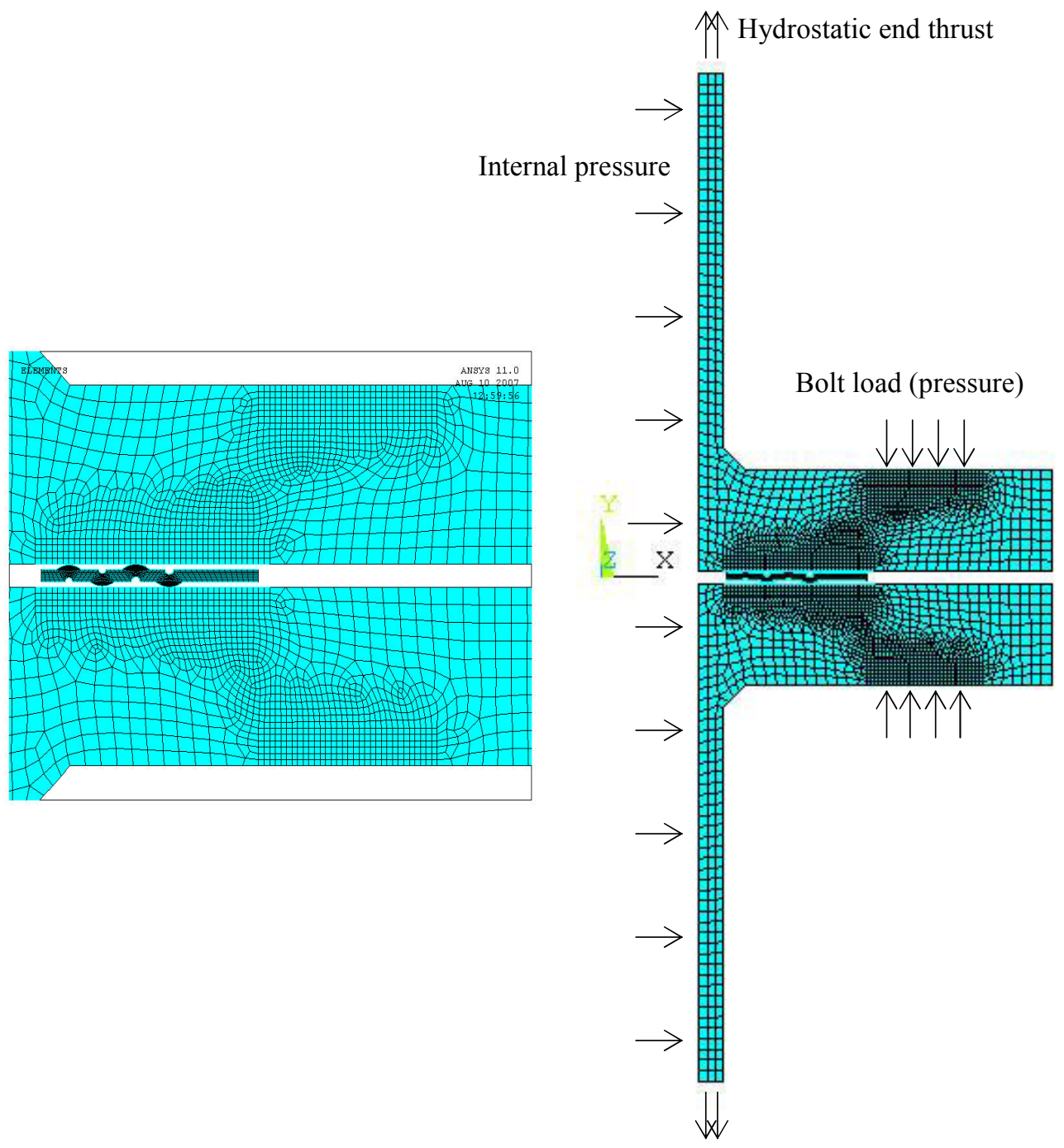

Fig. 3 Axisymmetric finite element model

There had to be a tradeoff between accuracy and efficiency, especially for smaller flange sizes (due to smaller number of bolts (4) only $45^{\circ}$ symmetry boundary conditions could be utilized). For bigger sizes with greater number of bolts however, 3D meshes may become feasible. Two dimensional axisymmetric model used in optimization is shown in Fig. 3.

We used the commercial finite element package Ansys to perform the analysis. Contact compatibility was enforced by using Augmented Lagrange algorithm. Solid elements capable of supporting plasticity, large deflection and large strain were used for modeling the flange and gasket. Sliding at the flange-gasket interaction was controlled by Coulomb friction model. Contact elements allowing Coulomb and shear stress friction were used at the flange-gasket interface. A coefficient of friction of 0.3 was assumed for interaction between flange and gasket lips, because of lack of data.

Strictly speaking, the flange material is inhomogeneous due to the discrete nature of bolt 
holes. But because the number of bolt holes for $25 \mathrm{~A}$ gasket flanges is only 4 , the reduction of stiffness is minor. That is why, we have ignored it here. However, when dealing with larger flange sizes, the difference in stiffness becomes considerable and in those cases, a procedure similar to that found in ASME code Section 8 (used to determine the effective material properties for perforated plates with holes) should be followed. The procedure is described in detail by Estrada et al ${ }^{(8)}$.

The total bolt load is smeared over the bolt-hole surface as a normal pressure, as shown in Fig. 3. The internal pressure is applied directly to the inner walls of pipe as well as flange inner surfaces. Hydrostatic end thrust is applied to the top of the pipe wall in the form of negative pressure.

In an axisymmetric analysis, the formulation of the element takes care of the boundary conditions in the circumferential direction. Therefore, the only constraint we have to provide is in the axial direction. The nodes at the top and bottom faces of the pipe are coupled with two spring elements (with minute stiffness) to prevent rigid body motion.

\subsection{Optimization results and discussion}

The data from FEM simulations based on Taguchi methodology was analyzed using regression analysis. Figs. 4 and 5 show the S/N plot for evaluation criterion of contact stress and contact area (contact length, since we performed a 2D analysis) respectively.

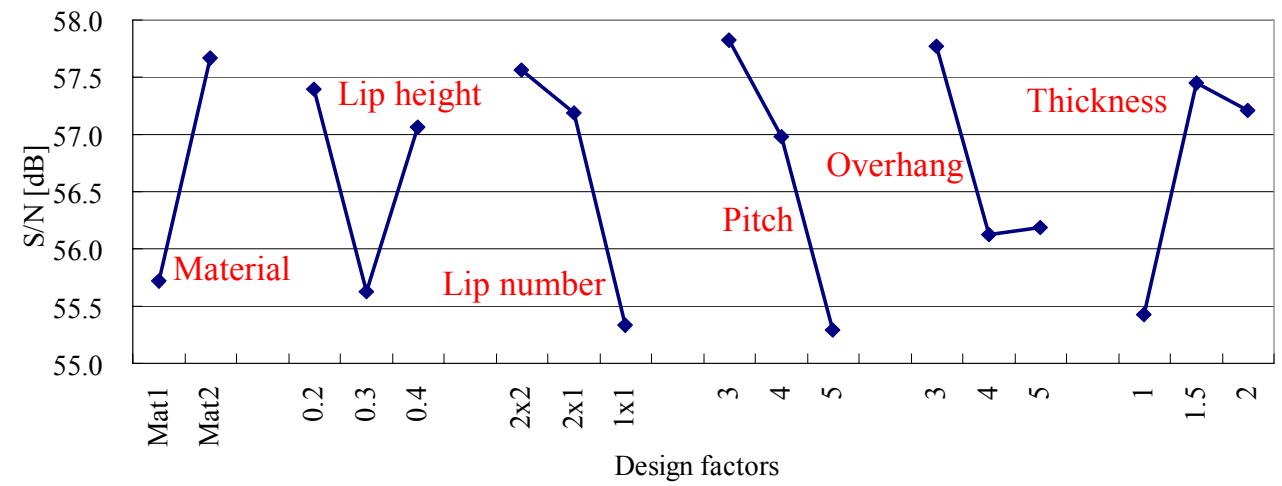

Fig. 4 S/N plot: Contact stress as evaluation criterion

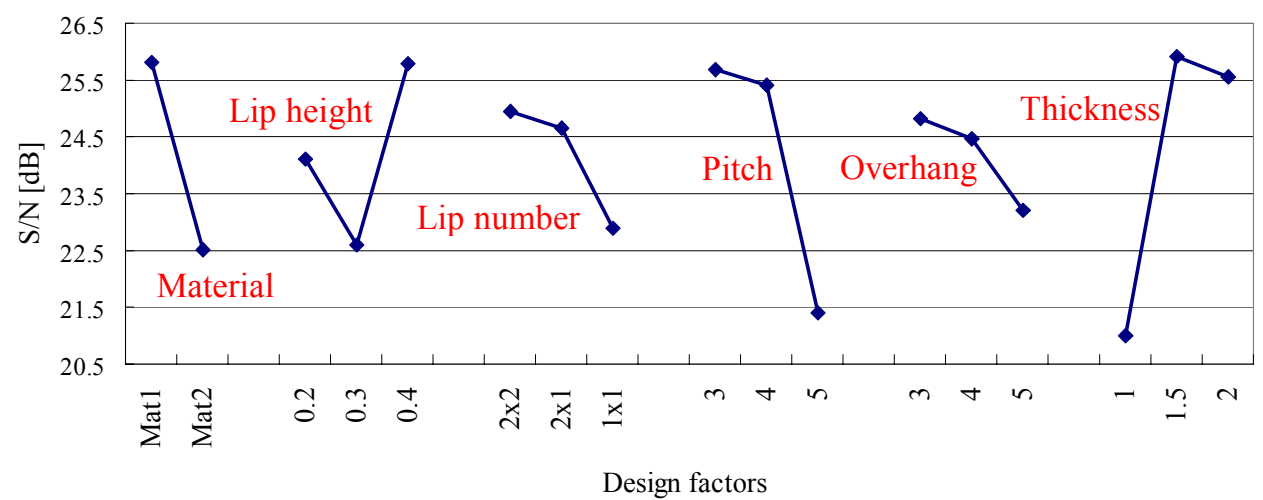

Fig. 5 S/N plot: Contact area as evaluation criterion

Sensitivity analysis of the design parameters was also carried out. Figs. 6 and 7 show the results of sensitivity analysis with respect to evaluation criteria of contact stress and 
contact area (contact length, since we performed a 2D analysis) respectively.

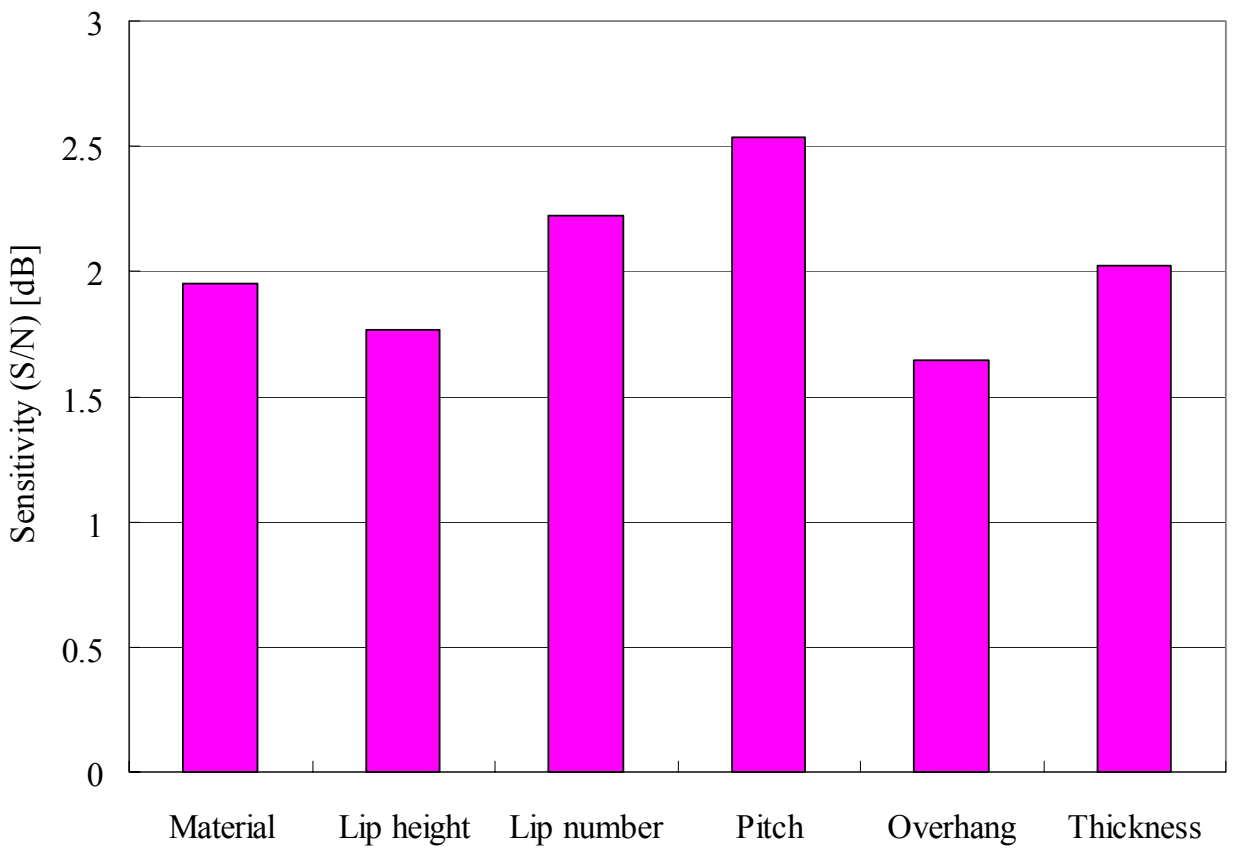

Design factors

Fig. 6 Sensitivity analysis: Contact stress as evaluation criterion

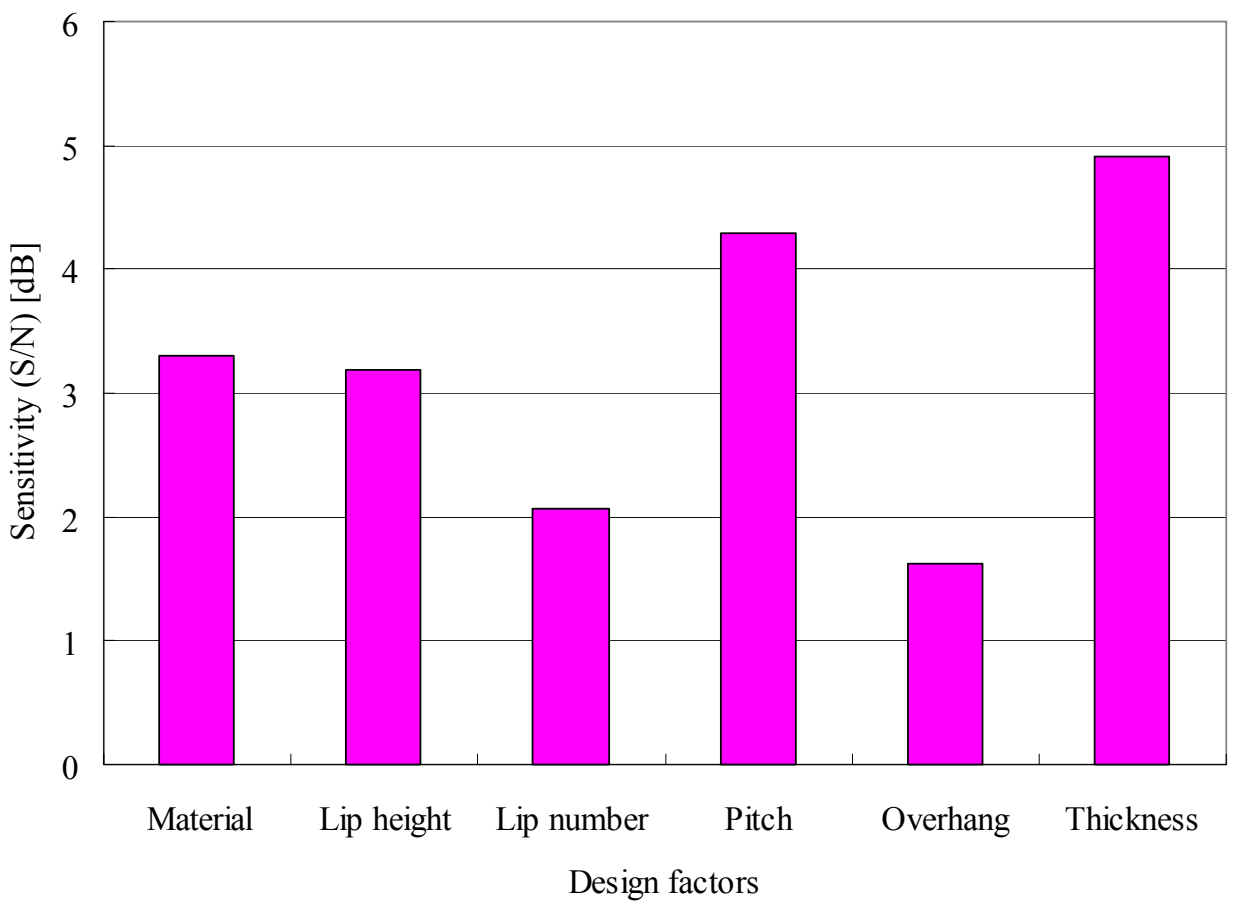

Fig. 7 Sensitivity analysis: Contact area as evaluation criterion

Optimum design can be picked directly from $\mathrm{S} / \mathrm{N}$ plots. Sensitivity analysis, on the other hand, tells us about the effect of each design parameter on the robustness of the system. The optimum levels for the two evaluation criteria are summarized in Table 5. 
Table 5 Optimum design levels

\begin{tabular}{|c|c|c|}
\hline \multirow{2}{*}{ Design factor } & \multicolumn{2}{|c|}{ Evaluation criterion } \\
\cline { 2 - 3 } & Contact stress & Contact area \\
\hline Material & Mat 2 & Mat 1 \\
\hline Lip height $\mathrm{h}[\mathrm{mm}]$ & 0.2 & 0.4 \\
\hline Lip number & $2 \times 2$ & $2 \times 2$ \\
\hline Pitch $\mathrm{p}[\mathrm{mm}]$ & 3 & 3 \\
\hline Overhang $\mathrm{OH}[\mathrm{mm}]$ & 3 & 3 \\
\hline Thickness $\mathrm{t}[\mathrm{mm}]$ & 1.5 & 1.5 \\
\hline
\end{tabular}

It is obvious that with the exception of material and lip height, both evaluation criteria suggest the same optimum conditions. Considering their respective yield strengths, the difference in optimum material is self-explanatory. As for the difference in lip height, inspection of the $\mathrm{S} / \mathrm{N}$ plot for contact stress shows that $0.4 \mathrm{~mm}$ is a close second after the optimum $0.2 \mathrm{~mm}$. This means that our formulation has yielded reasonable results and we can place confidence in our results.

The big question before us, then, was the selection of the evaluation criterion for experimental verification. This being a new kind of gasket; no guidelines in the form of past research, standard codes, or empirical rules, were available. For experimental verification, in the absence of a scientific basis, we decided to give precedence to the optimum design based on evaluation criterion of contact area. This decision was based on our assumption that a larger contact area is more beneficial than a larger contact stress as it can counter the roughness of the contact surfaces; because it can be argued that provided the contact stress is greater than internal pressure of the contained fluid, there should be no leaking. Thus performance of the optimum design based on contact area was verified experimentally.

\section{Experimental verification}

\subsection{Experimental setup}

Experimental testing was performed for the quantitative evaluation of the optimization results. Helium vacuum test, as specified by JIS Z $2330^{(9)}$ and JIS Z $2331^{(10)}$, was carried out for that purpose. Fig. 8 shows the schematic view of a vacuum type helium leak test machine.

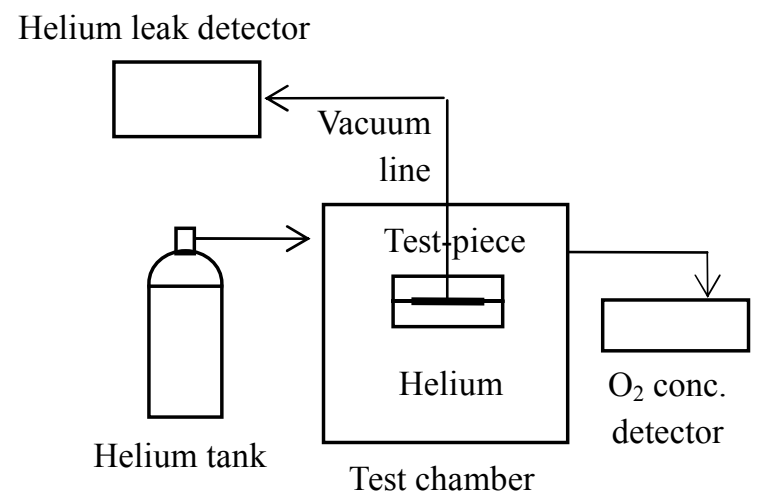

Fig. 8 Vacuum type helium leak test machine (schematic) 
The evacuated test object (gasketed flanges in our case) is placed in a test chamber that is filled with helium gas from a helium tank. Helium concentration inside the chamber is constantly monitored by an oxygen concentration sensor, and the test chamber is maintained at levels above $99 \%$ helium. Helium enters through all leaks present, into the evacuated test object and is sent to a helium detector by means of auxiliary (vacuum) pumps. The detector then indicates the leak rate, which can be converted to units of $\mathrm{Pa} \cdot \mathrm{m}^{3} / \mathrm{s}$.

\subsection{Experimental results}

The results of helium leak test, both before and after optimization, are shown in Fig. 9. "Unoptimized 1" and "Unoptimized 2" are the two best results of different designs based on variations of design variables in an arbitrary way (the other designs all yielding a leak rate of about $1.00 \mathrm{E}-03 \mathrm{~Pa} \cdot \mathrm{m}^{3} / \mathrm{s}$ even at $30 \mathrm{kN}$ preload). "Optimized" is the result of the design based on optimization performed by Taguchi method. It is evident that even at very low bolt preloads, the new design provides a marked improvement on the original trial and error designs.

It should be noted that the x-axis in Fig. 9 shows the nominal bolt preload set in the four bolts. The effects of noise, including the variation in preload of each of the four bolts, are included in these plots. Higher slope for "Optimized" design shows a higher functionality (less sensitivity to noise) and hence higher robustness.

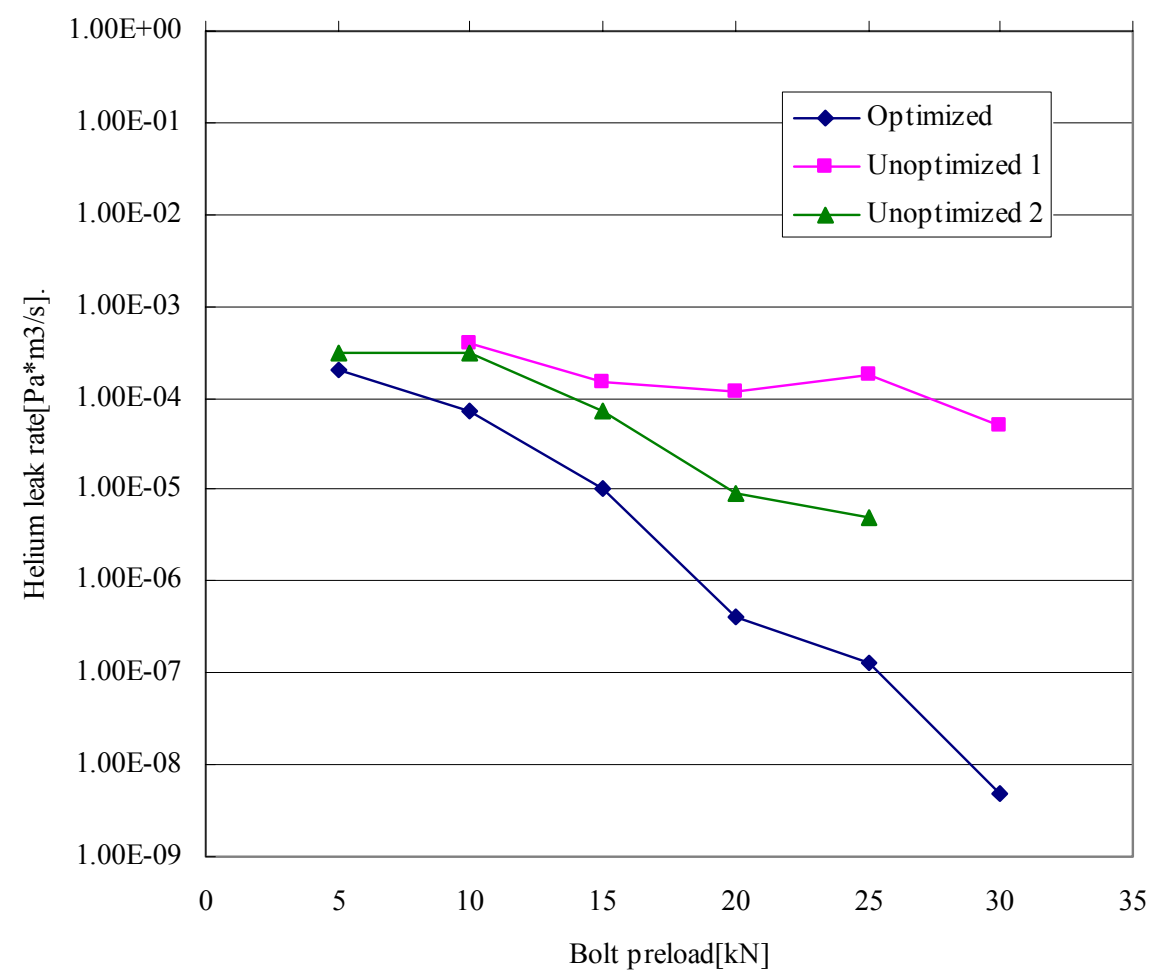

Fig. 9 Results of vacuum type helium leak test

\section{Conclusions}

A new kind of gasket (Super Seal Gasket) has been introduced. Because the approach taken in this study is based on mechanical design as opposed to the traditional approach of material development, there was need to optimize the different design parameters of the new gasket for leakage performance. Therefore, an optimization methodology based on the Taguchi method and the FEM was developed. As a test case, the design of a 25A sized industrial gasket was optimized. Helium leak test results reveal considerable improvement 
in sealing performance, thereby verifying the methodology developed. It is hoped, therefore, that the methodology developed here can be successfully extended to sizes other than $25 \mathrm{~A}$.

Results obtained are promising and it can be stated that material development is not the only approach towards the development of new, more effective gaskets. Also, Super Seal Gasket presented in this paper, has been shown to have potential for useful contribution in the sealing of flanged joints.

The effectiveness of Taguchi method to the problem of leaking has also been verified. The Taguchi methodology was utilized for designing FEM simulation runs. These simulations were effective in predicting the leaking performance, even though FEM cannot deal with the phenomenon of leaking directly. This was done by careful selection of evaluation criteria. The results justify the selection of contact area as a reasonable evaluation criterion. Owing to the effectiveness of FEM simulations, real hardware testing was used only for confirmation of optimization results, thereby saving considerable time and resources.

\section{Acknowledgement}

This research was funded by the Kyushu Bureau of Economy, Trade and Industry, agreement number 18G8023.

\section{References}

(1) Asahi newspaper, Economy section, 2005/9/26, p.7 (Japanese).

(2) Patent Cooperation Treaty: Appl. No. PCT/JP2005/19631 (Daiki Industries Co., Ltd.).

(3) Ranjit K. Roy, A Primer on the Taguchi Method, Society of Manufacturing Engineers, (1990), pp.211-224.

(4) Daniel E. Czernik, Gaskets: Design, Selection, and Testing, McGraw-Hill, (1996), pp. $98-104$

(5) John H. Bickford, Gaskets and Gasketed Joints, Marcel-Dekker, (1998), pp.524-532.

(6) Naoaki Noda, et al., Sealing performance of new gasketless flange, ASME J. Pressure Vessel Tech., 124, (2002), pp.239-246.

(7) Genichi Taguchi, et al., Robust Engineering, McGraw-Hill, (2000), pp.6-7.

(8) H. Estrada, et al., Strength and leakage finite element analysis of GFRP flange joint, Int. J. Pressure Vessels and Piping, 76, (1999), p. 548.

(9) JIS Z 2330, Standard recommended guide for the selection of helium leak testing, Japanese Standards Association, (1992).

(10) JIS Z 2331, Method of helium leak testing, Japanese Standards Association, (2006). 